\title{
Infezione da Helicobacter pylori e patologie correlate: nuove acquisizioni
}

\section{Giorgio Bertoni}

Servizio di Endoscopia Digestiva - Azienda Ospedaliera S. Maria Nuova - Reggio Emilia

\section{RELAZIONE}

L'argomento affidatomi è impegnativo soprattutto per la sua vastità. Negli ultimi anni, infatti, la ricerca sull'Helicobacter pylori $(\mathrm{Hp})$ si è sviluppata a $360^{\circ}$ verso quasi ogni possibile aspetto dello scibile patologico umano e sintetizzare questa massa di nuove conoscenze, spesso ancora incomplete, non è agevole.

Non vi è dubbio, comunque, che la scoperta, o meglio la riscoperta, dell' Helicobacter ha determinato una vera rivoluzione nella Gastroenterologia e nella Medicina in generale modificando il destino di un gran numero di pazienti così come il comportamento quotidiano di noi medici.

Pertanto, prima di passare ad illustrarvi le ultime nuove affascinanti conoscenze in materia sento il dovere di ricordare i nomi e i meriti di coloro che con le loro intuizioni e ricerche hanno reso possibile questo grande progresso della medicina.

Essi sono Robin Warren, anatomo-patologo, e Barry Marshall, gastroenterologo, entrambi semplici medici ospedalieri dell'anonima provincia Australiana. Prima di loro per quasi un secolo pressochè ogni patologo del mondo si era imbattuto in bacilli ricurvi non identificati sulla superficie mucosa gastrica senza attribuirvi alcun significato clinico in accordo con un dogma imperante della medicina che recitava: " Lo stomaco umano è un organo sterile al cui interno i germi trasportati dalla saliva e dagli alimenti vengono rapidamente distrutti dall'elevata acidità del succo gastrico. Perciò $i$ batteri osservati nello stomaco sono solo dei temporanei contaminanti incapaci di sopravvivere e di esercitare un ruolo patogeno". Furono Marshall e Warren i primi ad ipotizzare una stretta correlazione fra tali bacilli, la gastrite cronica attiva e la malattia ulcerosa peptica pubblicando due fondamentali lavori su Lancet nel 1983-84 (Lancet 1983;i:1273-5,Lancet 1984;i:1311-4). Ma Barry Marshall fece di più. Per dimostrare il terzo e quarto postulato di Koch - e cioè che l'inoculazione di tali germi in coltura pura era in grado di produrre la malattia nell'ospite umano e che gli stessi germi erano poi ritrovabili nelle lesioni così prodotte - bevve una coltura pura di Helicobacter dimostrando così che la sua mucosa gastrica in precedenza normale aveva sviluppato una forma di infiammazione acuta poi denominata gastrite da Helicobacter pylori .
Questi risultati furono rapidamente confermati da altri laboratori di tutto il mondo e da allora vi fu una vera e propria esplosione di studi sull'argomento che hanno raggiunto a tutt'oggi il numero di svariate migliaia generando numerose associazioni di studio e riviste mediche dedicate. Nel 1995 a Barry Marshall fu assegnato il premio Lasker per la ricerca medica che, come noto, segue per prestigio subito dopo il Nobel. Molti ritengono tuttavia che egli meriterebbe il Nobel.

\section{H. pylori e ulcera peptica}

La più formidabile evidenza della fortissima correlazione eziologica fra infezione da $\mathrm{Hp}$ e malattia ulcerosa peptica sta in 7 trials controllati randomizzati dimostranti che la sola terapia antibiotica eradicante, senza uso di farmaci antisecretori, è in grado di far cicatrizzare le ulcere gastriche e duodenali in misura uguale o superiore a quanto ottenibile con i più potenti farmaci antisecretori, ma con molte meno recidive a distanza (Lam SK et al: Gut 1997;41:43-8; Goh KL et al. Eur J Gastroenterol Hepat 1996;8:421-3 ;Hosking SW et al: Lancet 1994;343:508-10 ; Bayerdoffer E et al:Eur J Gastroenterol Hepat 1996;8:343-9; Sung JJ et al: N Engl J Med 1995;332:139-42; Massarrat S et al: Eur J Gastroenterol Hepat 1992; 4: 877-80; Xia H et al: Eur J Gastroenterol Hepat 1993;5:141-4).

Altri 8 studi osservazionali long-term hanno dimostrato che l'eradicazione dell' $H p$ riduce virtualmente a zero il rischio di recidiva emorragica a distanza nei pazienti con precedente ulcera gastrica o duodenale sanguinante (Pellicano R et al: J Clin Gastroenterol 2001;32:222-4; Vergara $\mathrm{M}$ et al:Eur J Gastroenterol Hepatol 2000;12:7337; Amendola $\mathrm{M}$ et al: Acta Gastroenterol Latinoam 1999;29:47-50; Macri $G$ et al: Am J Gastroenterol 1998;93:925-7; Riemann JF et al: Gastroeintes Endosc 1997;46:299-304; Jaspersen D et al: Gastroeintes Endosc 1995;41:5-7; Rokkas T et al:Gastrointest Endosc 1995;41:1-4; Graham DY et al: Scand J Gastroenterol 1993;28:93942). Mentre circa il 90\% delle ulcere duodenali sono H.pylori positive, e quindi eziologicamente correlate all'infezione, solo il $62 \%$ delle ulcere gastriche risulta H.pylori positivo. Del restante $38 \%$, ben $2 / 3$ sono correlate all'assunzione di FANS e solo poche hanno cause meno chiare -es. deficit di Epidermal Growth Factor salivare 
(EGF) o deficit dei recettori gastrici per l'EGF(Borody TJ et al. Am J Gastroenterol 1992;10:1403-7).

Dato quindi il potente effetto ulcerogenico dei FANS, indipendente dall'Helicobacter, e dato il crescente consumo di questi farmaci, non è pensabile di ottenere la scomparsa completa della malattia ulcerosa nella popolazione generale solo attraverso l'eradicazione dell' $H p$.

Ciò nonostante, la sistematica campagna di eradicazione operata negli ultimi 10 anni sugli individui infetti ha portato a un rilevantissimo successo, sia clinico che economico, sulla malattia ulcerosa. Dalla revisione comparativa dei dati di attività del nostro Servizio di Endoscopia Digestiva, riferita ad analoghi periodi campione del 1991 e del 2002 , risulta una riduzione dell' $80 \%$ nelle diagnosi endoscopiche di ulcera duodenale (82 soli casi nei primi 4 mesi del 2002 versus 395 nei primi 4 mesi del 1991) e del 45,5\% delle ulcere gastriche (78 casi versus 143) per un complessivo risparmio comparativo di circa 1200 gastroscopie l'anno.

Ciò, purtroppo, non si è tradotto in un calo delle richieste complessive di endoscopie, e quindi delle liste di attesa per i pazienti, a causa dell'inesorabile concomitante aumento delle richieste per altre indicazioni quali la MRGE e le patologie colorettali. E' tuttavia evidente che se non si fosse ottenuto un così importante successo terapeutico nei confronti della patologia ulcerosa la situazione in termini di sovraccarico di richieste, di ricoveri e di interventi d'urgenza per complicanze ulcerose sarebbe ben più critica dell'attuale.

\section{H. pylori e cancro gastrico}

Già nel 1994 lo IARC (International Agency for Research on Cancer) ha classificato l'Hp come agente cancerogeno di classe $\mathrm{A}$, inserendolo cioè nella stessa lista dei più potenti cancerogeni conosciuti quali il fumo di tabacco e le radiazioni ionizzanti (IARC: Lyon,1994;61:177241).

Vari studi hanno infatti evidenziato un associazione lineare fra infezione da $H p$, metaplasia intestinale e cancro gastrico (Valle J et al: Curr Opin Gastroenterol 1997;13:35-9).

Inoltre, quando si paragona una popolazione con un tasso di infezione del $100 \%$ con una non infetta si osserva un rischio di cancro 6 volte maggiore (Eurogast Study Group: Lancet 1993;341:1359-62) Tuttavia le variazioni geografiche nella prevalenza del cancro non possono essere spiegate esclusivamente con la prevalenza dell' $\mathrm{Hp}$. Alcuni studi hanno infatti dimostrato che un'alta prevalenza di gastrite $\mathrm{Hp}+$ non è necessariamente predittiva di un'alta prevalenza di cancro gastrico (Gasbarrini G et al: Ital J Gastroenterol
Hepatol 1998;30:5-8). La patogenesi del cancro gastrico deve perciò ritenersi multifattoriale e l'Hp appare essere un importante, ma non l'unico, fattore implicato (Valle $\mathrm{J}$ et al: Curr Opin Gastroenterol 1997;13:35-9).

Negli ultimi anni molta ricerca è stata rivolta verso la tossina Cag A, prodotta da circa il $60 \%$ dei ceppi di $\mathrm{Hp}$. La Cag A è una proteina immunomodulante che aiuta il batterio a meglio difendersi dagli attacchi del sistema immunitario dell'ospite (Allen LA et al: J Exp Med 200;191:1158). Questa tossina è stata collegata ad un aumentato rischio di sviluppare gastrite atrofica, metaplasia intestinale e ulcera peptica (Kuipers EJ et al: J Natl Cancer Inst 1995;87:1777-80 ; Cover TL et al: J Clin Microbiol 1995;33:1496-500). Alcuni studi hanno anche osservato una correlazione fra presenza della tossina e rischio di cancro gastrico (Parsonnet J et al: Gut 1997;40:297-301; Kuipers EJ et al: J Natl Cancer Inst 1995;87:1777-80).

Tuttavia, lo studio multinazionale europeo EUROGAST, così come altre recenti indagini epidemiologiche condotte in Cina, Giappone ed Estonia non hanno evidenziato alcuna differenza nella prevalenza di sieropositività per Cag $\mathrm{A}$ fra $\mathrm{i}$ soggetti con o senza cancro (Eurogast Study Group: Lancet 1993;341:1359-62; Kikuchi S et al: Am J Gastroenterol 1999;94:3455-59; Klamaas K et al:Int J Cancer 1996;67:1-5 ; Mitchell HM et al: Am J Gastroenterol 1996;91:1785-8).

Lo studio Giapponese, in particolare, ha osservato un identico potenziale cancerogeno fra i ceppi CagA positivi e negativi (Kikuchi $\mathrm{S}$ et al: Am J Gastroenterol 1999;94:3455-59). Al momento, quindi, il significato cancerogeno della sieropositività per $\mathrm{Cag} \mathrm{A}$ rimane quantomeno incerto. Le due ovvie conclusioni che si devono trarre ai fini della prevenzione del cancro sono che: mentre non possiamo manipolare la predisposizione genetica dei pazienti nello sviluppare l'una o l'altra patologia da $H p$, possiamo invece manipolare l'infezione stessa Occorre cioè eradicare l'infezione e indipendentemente dalla sieropositività $\mathrm{o}$ meno per il Cag A. Ciò, beninteso, non significa eradicare in massa tutta la popolazione infetta anche se asintomatica, ma focalizzarsi invece sui soggetti a rischio significativo. Cioè in accordo con le recenti Guidelines di Maastricht (The Maastricht 2000 Consensus Report - Aliment Pharmacol Ther 2002;16:167-80) i pazienti con familiarità per $\mathrm{K}$ gastrico, anche se asintomatici, $\mathrm{i}$ soggetti con alterazioni importanti dell'istologia mucosa gastrica (cioè atrofia, metaplasia intestinale, erosioni) e i soggetti con precedente resezione per $\mathrm{K}$ gastrico o con pregresse patologie preneoplastiche (es. adenomi gastrici) 
Negli ultimi anni vari studi hanno evidenziato che l'atrofia gastrica (AG) e la metaplasia intestinale (MI) non sono condizioni croniche irreversibili come un tempo creduto, ma che dopo l'eradicazione dell' $\mathrm{Hp}$ possono nettamente migliorare se non completamente regredire.

Cayla et al. per primi dimostrarono che a distanza di 4 anni dall'eradicazione l' AG e la MI regredivano completamente nel $75 \%$ dei soggetti e che $\mathrm{i}$ follicoli iperplastici (condizione preliminare per lo sviluppo del linfoma MALT) scomparivano in tutti i pazienti gia' dopo 2 anni (Cayla R et al. Gut 1995;37 suppl 2:A152).

Questi risultati sono stati recentemente confermati da altri autori Giapponesi, Finlandesi e Italiani Ito $\mathrm{M}$ et al. Aliment Pharmacol Ther 2002;16:1449-5; Kokkola A et al. Aliment Pharmacol Ther 2002;16:15-30; Tucci A et al. Am J Gastroenterol 1998; 9:1425-31.

Da tutto ciò ne consegue che l'eradicazione dell' $H p$ rappresenta, con ogni probabilità, il miglior modo per abbattere la prevalenza nella popolazione di questa malattia ad altissima mortalità. Per dimostrare questo assunto sono stati avviati studi prospettici su grandi casistiche di pazienti ma $\mathrm{i}$ dati conclusivi di tali studi non saranno disponibili, per ovvie ragioni, prima di vari anni.

\section{H. pylori e MALT linfoma gastrico (Mucosal-Associated Lymphoid Tumor)}

Molto più raro del carcinoma $(\sim 5 \%$ di tutti $\mathrm{i}$ tumori maligni gastrici) ma con un'alta prevalenza in alcune aree del nord-est Italiano e della Svizzera. Il 70-80\% dei MALT linfomi a basso grado di malignità regredisce completamente con la semplice eradicazione dell'Hp: Sepulveda AR et al. Helicobacter 2002;7:37-42; Lacy B et al. J Nutr 2001;131:2789S-93S; Morgnier A et al. Gastroenterol Clin NA 2000;29: 503-607; Doglioni C et al. Lancet 1992;339:834-5). Fattori predittivi di mancata risposta alla terapia eradicante sono: alto grado di malignità traslocazione $(11 ; 18)(\mathrm{q} 21 ; \mathrm{q} 21)$ nelle cell. Tumorali; presenza di invasione sottomucosa alla EUS; presenza di linfonodi perigastrici interessati; presenza di concomitanti malattie autoimmuni: Liu $\mathrm{H}$ et al. Lancet 2001;357:39-40 ;Sepulveda AR et al. Helicobacter 2002;7:37-42; Lacy B et al. J Nutr 2001;131:2789S-93S; Raderer S et al. Ann Oncol 2001;12:937-9

Anche nei casi responsivi alla terapia, comunque, l'atteggiamento clinico deve essere improntato a prudenza ed una stretta sorveglianza clinica è mandataria.

Comunque, il 90\% dei MALT linfomi regrediti con l'eradicazione rimane in remissione clinicoistologica completa e in apparenza permanente.
Anche se ulteriori, più ampi studi sono necessari per affermare che questi pazienti sono definitivamente guariti, i risultati della terapia eradicante su questa forma tumorale appaiono a dir poco entusiasmanti. Neubauer A et al. J Natl Cancer

Inst 1997;89:1350-5; Weber DM et al. Gastroenterology 1994;107:1835-8;Savio A et al. Gut 1997;37 (suppl 2) A246.

\section{H. pylori e polipi iperplastici}

L'esistenza di una stretta correlazione fra $\mathrm{Hp}$ e polipi gastrici iperplastici è stata segnalata fin dai primi anni 90 (Veereman $G$ et al. Am J Gastroenterol 1990;85:1395-7; Saito Y et al. Eur J Gastroenterol Hepatol 1992;4 :S89-92). I dati più rilevanti vengono però da due studi prospettici, di cui uno controllato randomizzato, indicanti che l'eradicazione induce la regressione endoscopica completa dei polipi nel 40-80\% dei pazienti entro 15 mesi dalla fine della cura ( Ohkusa $\mathrm{T}$ et al. Ann Int Med 1998;129:712-1; Ljubicic N et al. Eur J Gastroenterol Hepatol 1999; 11:727-30).

Perciò, anche se le evidenze attuali sono da condiderarsi ancora non definitive, occorre considerare, nell'iter terapeutico di questi pazienti, l'opportunità dell'eradicazione.

\section{H. pylori e adenomi gastrici}

Esiste un solo studio non randomizzato su 64 pazienti dei quali 32 sottosposti ad eradicazione e 32 non trattati (Saito K et al: Gastroeintest Endosc 2000;52:27-32). Durante i due anni di sorveglianza dello studio il $12,5 \%$ dei pazienti non trattati sviluppò all'interno dell'adenoma un carcinoma iniziale mentre nessuno dei pazienti eradicati sviluppò il cancro. Questo studio rappresenta perciò un ulteriore supporto all'importanza di eradicare i pazienti per prevenire il carcinoma gastrico.

\section{H. pylori e gastropatia a grosse pliche/M. di Menetrier}

Esiste una stretta correlazione fra infezione da $H p$, gastropatia a grosse pliche (cioè pliche di oltre $1 \mathrm{~cm}$ di spessore) e la gastropatia a grosse pliche con severa ipoproteinemia (cosiddetta Malattia di Menetrier) (Avunduk C et al. Am J Gastroenterol 1995;90:1969-73; Di Vita G et al: Dig Dis Scie 2001;19:179-83; Madsen LG et al: Dig Dis Scie 1999;44:2307-12 ; Badov D et al: Am J Gastroenterol 1998; 93:1976-9). L' Hp è infatti costantemente associato a questa patologia e la sua eradicazione determina sia la scomparsa della flogosi mucosa con normalizzazione dello spessore plicale in tutte le forme di semplice ipertrofia ( Avunduk C et al. Am J Gastroenterol 1995;90:1969-73) sia la regressione anche dell'ipoproteinemia nella M. di Menetrier dell'adulto e del bambino (Di Vita G et al: Dig Dis Scie 2001;19:179-83; Madsen LG et al: Dig Dis Scie 
1999;44:2307-12; Badov D et al: Am J Gastroenterol 1998;93:1976-9; Yamada M et al. Eur J Ped 1997;156:182-5).

\section{H. pylori e anemia ferro priva}

L'anemia ferro priva $\mathrm{Hp}$-correlata (ovviamente non associata a sanguinamento dimostrabile in sede digestiva o extradigestiva) non riguarda solo le donne in pre-menopausa, ma interessa anche i bambini e le ragazze adolescenti (ovviamente il sesso femminile favorisce la comparsa dell'anemia a causa delle concomitanti perdite mestrua1i). Konno $M$ et al. J Ped Gastroenterol 2000;31:52-6; Choe YH et al. Acta Pediatr 2000 89:154-7. Nei vari studi effettuati l'eradicazione dell' $H p$ ha determinato una rapida e stabile regressione dell'anemia in tutti i pazienti trattati, sia adulti che bambini che adolescenti (Annibale $B$ et al. Ann Int Med 1999;131:668-72 ; Sugyiyama $T$ et al. Intern Med 2002;41:491-4; Choe $\mathrm{H}$ et al Helicobacter 1999;4:135-9; Konno $\mathrm{M}$ et al. J Ped Gastroenterol 2000;31:52-6). Data la concordanza delle evidenze fin qui pubblicate è verosimile ritenere che l'anemia ferro priva idiopatica $\mathrm{Hp}+$ sia una delle patologie maggiormente candidate ad essere inserita fra le nuove indicazioni al trattamento eradicante nelle prossime linee-guida dell' EHPSG. Stanno anche emergendo evidenze, per quanto meno forti, di una correlazione fra anemia megaloblastica, deficit di vit.B12 e infezione da $H p$. Il $56 \%$ di questi soggetti ha l'infezione e il $40 \%$ di essi ha mostrato una regressione stabile dell'anemia con l'eradicazione ( Kaptan $\mathrm{K}$ et al. Arch Int Med 2000;160:1349-53; Serin E et al. Helicobacter 2002;7:337-41).

\section{H. pylori e alitosi}

Può sembrare inopportuno soffermarsi su una patologia considerata così "leggera", ma occorre ricordare che nelle forme gravi l'alitosi può causare seri problemi psicologici e limitare la stessa vita di relazione. Due studi prospettici hanno studiato la correlazione fra alitosi, infezione da $H p$ e risposta alla terapia eradicante su un totale di 208 pazienti. Uno dei due studi ha anche, per la prima volta, indagato oggettivamente il problema attraverso il dosaggio dei composti odorosi sulfidici nel respiro (Ierardi E et al. Dig Dis Scie 1998;43:2733-7; Serin E. Eur J Intern Med 2003; 14:45-8).

In questi due studi, l'alitosi ha mostrato un netto decremento in tutti i pazienti eradicati rimanendo invece invariata in quelli non eradicati. Il dosaggio dei composti sulfidici ha rivelato una loro caduta, al di sotto del cut-off di rilevabilità, nei pazienti eradicati ma non in quelli con infezione persistente. La conclusione di questi studi è, che nei soggetti $H p$ positivi, l'alitosi è probabilmente legata all'attività microbica putrefattiva del batterio in cavità gastrica e che $\mathrm{l}^{\prime} \mathrm{Hp}$ va perciò ricercato e trattato in questi soggetti ( Ierardi E et al. Dig Dis Scie 1998;43:2733-7; Serin E. Eur J Intern Med 2003;14:45-8).

\section{H.pylori e assunzione di FANS}

Si tratta di un argomento complesso e i molti studi fin qui condotti hanno portato a risultati spesso contradditori anche perché i FANS costituiscono, di per sé, un potente fattore ulcerogenico indipendente dall' $H p$ (Hawkey CJ et al. Gut 2002;51:336-43; Lanza FL. Am J Gastroenterol 1998;93:2037-45).

In sintesi l'evidenza attuale indica che:

1. l'Hp contribuisce ad incrementare il rischio di ulcera all'inizio di un trattamento con FANS anche se

2. i FANS sono probabilmente i maggiori responsabili di gran parte delle ulcere osservate in coloro che li assumono da lungo periodo

3. l'Hp contribuisce al sanguinamento ulceroso nei pazienti che assumono cronicamente aspirina a basso dosaggio nei pazienti $H p+$ con storia di ulcera peptica, e che assumono aspirina a basso dosaggio

4. l'eradicazione batterica ha un effetto preventivo sul sanguinamento comparabile alla somministrazione cronica di omeprazolo (Hawkey CJ et al. Gut 2002;51:336-43; Lanza FL. Am J Gastroenterol 1998;93:2037-45).

\section{H. pylori e anticoagulanti orali}

La letteratura è limitatissima. E' consuetudine clinica richiedere un controllo endoscopico nei pazienti anticoagulati con sintomatologia gastralgico/dispeptica o con pregressa storia di ulcera, ma poco è noto circa $i$ pazienti asintomatici che possono sviluppare emorragie severe "a ciel sereno".

In un recente studio prospettico da noi effettuato su 216 pazienti asintomatici anticoagulati, 120 (56\%) risultarono $\mathrm{Hp}+$ al C13 urea breath test. Di questi 86 furono sottoposti a gastroscopia e 69 $(80 \%)$ evidenziarono patologie digestive di varia natura di cui 29 (cioè il $36,2 \%$ del totale dei positivi) direttamente correlate all' $\mathrm{Hp}$ e potenzialmente emorragiche. Fra queste furono osservate 7 $\mathrm{UD}, 2 \mathrm{UG}$ e $2 \mathrm{~K}$ gastrici asintomatici (Bertoni G, Tincani E et al. Am J Med 2000;108:165-7).

Le conclusioni sono che lesioni gastroduodenali $\mathrm{Hp}$ correlate e potenzialmente emorragiche sono riscontrabili in oltre $1 / 3$ dei pazienti anticoagulati asintomatici e che l' $H p$ dovrebbe pertanto essere sistematicamente ricercato ed eradicato in questi pazienti ad alto rischio.

\section{Eradicazione dell' $H p$ e MRGE}

Negli ultimi anni sono stati sollevati timori che l'eradicazione dell'Helicobacter possa favorire 
l'insorgenza di una MRGE e ciò ha generato un intenso dibattito scientifico. Vari studi recenti hanno tuttavia ben definito i termini del problema che possono essere così riassunti:

- l'eradicazione dell'Hp non influenza il decorso di una pre-esistente MRGE (O'Connor HJ et al. Aliment Pharmacol Ther 1999;13:11727; Axon AT et al. Gastroenterology 1999;116:A117)

- l'eradicazione dell'Hp migliora il decorso di una MRGE associata ad ulcera duodenale ( Dent J et al. Aliment Pharmacol Ther 2001;15 -suppl 1:16-21).

- nella grande maggioranza dei soggetti senza pre-esistente MRGE l'eradicazione dell' $H p$ non favorisce lo sviluppo di un reflusso patologico. Esiste tuttavia un piccolo sottogruppo di pazienti $(<10 \%)$ con gastrite del corpo, in cui l'eradicazione può associarsi alla comparsa di un reflusso patologico (Dent $\mathrm{J}$ et al. Aliment Pharmacol Ther 2001;15 -suppl 1:1621; Hamada $\mathrm{H}$ et al. Aliment Pharmacol Ther 2000;14:729-35). Anche in questi pazienti però è stato dimostrato che l'eradicazione non ha alcuna influenza diretta sullo sviluppo del reflusso ma semplicemente slatentizza un reflusso già pre-esistente, mascherato dai bassi livelli di secrezione acida indotti dall'infezione stessa. In ogni caso, comunque, l'eventuale reflusso si sviluppa sempre in forma lieve o paucisintomatica (Veldhuyzen SJO etal. Dig Liver Dis 2001;33:647-52).

Il panel di esperti dell' EHPSG ha pertanto concluso che "le preoccupazioni circa un rapporto negativo fra eradicazione dell' $H p$ e MRGE sono largamente infondate e non devono prevenire l'adozione della terapia eradicante negli idonei gruppi di pazienti" (The Maastricht 2000 Consensus Report - Aliment Pharmacol Ther 2002; 16:167-80).

\section{Infezione da $\boldsymbol{H p}$ e Dispepsia Non Ulcerosa}

I complessi rapporti fra infezione da Hp e dispepsia non ulcerosa verranno trattati nella prossima relazione del Dott. Fornaciari.

Gli aspetti più nuovi e sorprendenti che stanno emergendo negli ultimi anni sono invece quelli fra H.pylori e patologie extradigestive e cioè:

- Cardiopatia ischemica

- Ictus cerebri

- Orticaria cronica idiopat.

- Prurito cronico

- Acne rosacea

- M. di Raynaud

- Artrite reumatoide

- Cefalea emicranica

- Porpora trombocit.idiop.

- Gammop. monoclonale
- Glaucoma ad angolo ap.

- Infertilità

- Deficit staturale

- Ridotta crescita fetale

- Iperemesi gravidica

- Bronchite cronica

- Litiasi biliare

- Cancro del pancreas

- Epidemie di colera nel $3^{\circ}$ mondo

\section{H.pylori e Cardiopatia ischemica}

La coronaropatia ischemica è la principale causa di morte nei paesi occidentali. C'è ora convincente evidenza che l'aterosclerosi è molto più che un semplice accumulo intravascolare di lipidi e che un'infiammazione acuta e cronica dell'intima arteriosa influenza l'iniziazione, la progressione e l'instabilità delle placche ateromasiche, oltre a favorire la re-stenosi coronarica dopo trattamento. Infatti, tutti i ben noti fattori di rischio per l'aterosclerosi (ipertensione, fumo,dislipidemia, diabete, iperomocisteinemia) non possono spiegare più del $60 \%$ dei casi di malattia. ( Kahan $\mathrm{T}$ et al. Coron Artery Dis 2000;11:523-6; Di Tano G et al. Ital Heart J 200;1:1576-81; Mawhorter SD et al. Cleve Clin J Med 2001; 68:449-58).

Vari di studi hanno evidenziato una correlazione, o una forte correlazione, fra infezione da $\mathrm{Hp}$ e malattia coronaria (Osawa $\mathrm{H}$ et al. Cardiology 2001;95:14-9; Kahan T et al. Coron Artery Dis 2000;11:523-6; Hara H et al. Clin Chim Acta 2001;313:87-94; Ossei-Gerning $\mathrm{N}$ et al. Cardiovasc Res 1997;35:120-4), ma altri studi hanno smentito tale correlazione (Smieja M et al. Circulatio 2003;107:251-7; Zhu J et al. Am J Cardiol 2002;15:155-8; Whincup PH et al. Heart 1996;75:568-72). Il meccanismo patogenetico con cui l'Hp favorirebbe il danno coronarico è stato attribuito ad un incremento dei livelli di proteina C reattiva (Sanderson J1 et al. J AM Coll Cardiol 1998;32:35-41) e ad una diminuzione nella concentrazione serica di HDLC (Takashima $\mathrm{T}$ et al. Helicobacter 2002;7:86-90). È stato affermato che solo i ceppi Cag A + si associano ad un aumentato rischio di coronaropatia (Gun $\mathrm{M}$ et al. Heart 2000;84:267-71; Franceschi $F$ et al. Circulation 2002;106:430-4) ma non tutti gli studi hanno confermato questo dato (Murray LJ et al. Atherosclerosis 2000;149:379-85). È stato anche segnalato che l'Hp potenzia il ruolo patogeno svolto dalla Clamydia pneumoniae, altro agente microbico fortemente sospettato nella patogenesi del danno coronarico (Schumaker A et al. Clin Microbiol Infect 2002;8:654-61) e, infine, dopo vari tentativi andati a vuoto il DNA dell'Helicobacter è stato isolato all'interno delle placche ateromasiche coronariche di pazienti con precedente infarto miocardico o angina instabile 
con ciò supportanto l'ipotesi di una correlazione diretta fra l'infezione e la progressione/instabilità delle placche ateromasiche (Kowalski $\mathrm{M}$ et al: Dig Liver Dis 2002;334:398-402). Sono inoltre disponibili due trials di terapia eradicante nei pazienti con severa cardiopatia ischemica. Il primo studio ha dimostrato una riduzione statisticamente significativa nel rischio di re-stenosi coronarica dopo PCA nei soggetti eradicati rispetto al gruppo di controllo (Kovalski $\mathrm{M}$ et al. Dig Liv Dis 2001;33:222-9). Il secondo studio ha osservato una riduzione del $36 \%$, statisticamente significativa, degli end points clinici (morte e riammissione ospedaliera per nuovo episodio coronarico acuto) nei soggetti infartuati trattati con antibiotici, ma sconcertantemente questo effetto è risultato indipendente dalla sieropositività per $\mathrm{Hp}$ o Clamydia pneumonite. (Stone $\mathrm{AF}$ et al. Circulation 2002;106:1219-23). Nel complesso occorre purtroppo concludere che la varietà $\mathrm{e}$ complessità dei fattori eziologici coinvolti, la possibilità di molteplici fattori confondenti e la disomogeneità delle casistiche studiate, non ci consente di trarre ancora conclusioni definitive.

Quello che invece sta emergendo in maniera abbastanza chiara è il ruolo del cosiddetto "fardello infettivo individuale" come nuovo importante fattore di rischio per cardiopatia ischemica.Per fardello infettivo si intende il complesso delle risposte sierologiche anticorpali dell'individuo nei confronti di molteplici agenti patogeni quali, oltre all' $\mathrm{Hp}$ e alla Clamydia pneumoniae, il Citomegalovirus, il virus di Epstein-Barr, gli Herpes simplex virus 1 e 2, l'Hemophilus influenzae e il virus dell'epatite $\mathrm{A}$. La sieropositività per 5 o più di questi patogeni accresce significativamente il rischio infartuale indipendentemente da tutti gli altri classici fattori di rischio (Prasad A et al.Circulation 2002;106:184-9; Rupptrecht HJ et al. Circulation 2001;104:25-31 ; Horne BD et al. Am Heart J 2002; 144:491-50).

\section{H.pylori e ictus cerebrale}

Sette studi caso controllo hanno indagato il rapporto fra infezione da $H p$ e ictus ischemico. Cinque studi hanno osservato una prevalenza significativamente maggiore di infezione nei casi colpiti rispetto ai controlli sani (Ponzetto A et al. Hepatogastroenterol 2002;49:631-4; Grau AJ et al. J Neurol Sci 2001;186:1-5; Markus HS et al. J Neurosurg Psychiatry 1998;64:104-7) ma due di questi solo relativamente ai ceppi Cag A+ (Majka $\mathrm{J}$ et al. Med Sci Monit 2002;10:675-84; Pietroiusti A et al. Circulation 2002;106:580-4).

Uno studio ha osservato livelli di colesterolo totale, colesterolo LDL, fibrinogeno e IL-8 significativamente maggiori nei soggetti infetti. Sei mesi dopo l'eradicazione dell' $H p$, i livelli plasmatici di tutti questi fattori si riducevano significativamente (Majka J et al. Med Sci Monit 2002;10:675-84). Questi dati non consentono di affermare che esiste una correlazione certa fra $\mathrm{Hp}$ e ictus ischemico ma sono comunque in linea con quanto già detto a proposito della cardiopatia ischemica.

\section{H.pylori e Orticaria cronica idiopatica/Prurito cronico}

Le prime segnalazioni di regressione dell'orticaria cronica dopo eradicazione dell' $\mathrm{Hp}$ risalgono al 1995 (Bohmeyer J et al. Hautarzt 1996;47:106-8; Tebbe B et al. J Am Acad Dermatol 1996;34:6856). Negli ultimi anni dozzine di studi hanno chiarito molti aspetti del problema ma non hanno ancora fornito quella risposta definitiva che tutti attendono. Cinque studi prospettici controllati e un piccolo trial randomizzato doppio-cieco versus placebo hanno riportato una remissione completa o parziale dell'orticaria nel $60-88 \%$ dei pazienti dopo l'eradicazione ( Sakurane $\mathrm{M}$ et al: J Dermatol 2002;29:23-7; Kandyl R et al. J Cut Med Surg 2002;6:103-8; Shiotani A et al. Helicobacter 2001;6:60-5; Wedi B et al. Int Arch Allergy Immunol 1998;116:288-94; Di Campli C et al. Dig Dis Sci 1998;43:1226; Gaig P et al. Allergol Immunopathol 2002;30:255-8).

Altri studi hanno riportato solo un più modesto $10-33 \%$ di risposte (Dauden $\mathrm{E}$ et al. Int $\mathrm{J}$ Dermatol 2000;39:446-52; Schnyder B et al. Int Arch Allergy Immunol 1999;119:60-3; Valsecchi $\mathrm{R}$ et al. Acta Dern Venereol 1998;78:440-2). Remissioni complete o parziali post-eradicazione sono state osservate anche nel $30-73 \%$ dei soggetti affetti da prurito cronico, da dermatite nummulare e da prurigo cronica multiformis (Sakurane M et al: J Dermatol 2002;29:23-7; Kandyl R et al. J Cut Med Surg 2002; 6:103-8; Shiotani A et al. Helicobacter 2001; 6:60-5; Wedi B et al. Int Arch Allergy Immunol 1998; 116:28894). La patogenesi delle lesioni sembra mediata dallo sviluppo di autoanticorpi diretti contro la frazione Fc delle cellule istamino produttrici, anticorpi probabilmente generati come risposta verso antigeni lipopolisaccaridici dell' Helicobacter. (Gala-Ortiz G et al. Ann Allergy Asthma Immunol 2001;86:696-8; Greaves MW et al. Int Arch Allergy Immunol 2002; 127:3-9). Sono chiaramente necessari ulteriori studi prima di poter raccomandare il testaggio sistematico dell' $H p$ in tutti pazienti affetti da OCI o PCI. Tuttavia, nei soggetti non rispondenti alla terapia tradizionale, la ricerca e l'eradicazione del batterio appaiono proponibili.

\section{H.pylori e acne rosacea}

Anche per l'acne rosacea i dati di studio sono contradditori con positività di risposte che varia- 
no addirittura dal $96 \%$ a zero (Szlachcic A. J Eur Acad Dermatol Venereolog 2002;164:328-33; Utas S et al. J Am Acad Dermatol 1999;40:433-5; Herr H et al. J Korean Med Sci 2000;15:551-4; Bamford JT et al. Arch Dermatol 1999;659-63).

Il mondo dei dermatologi appare quindi spaccato fra sostenitori e detrattori e ogni conclusione appare prematura. Tuttavia, il fatto che le papulopustole possano migliorare anche solo con pomate locali a base di antibiotici fa supporre che l'effetto benefico della terapia eradicante possa in realtà derivare solo dall'azione degli antibiotici sistemici su altri germi cutanei (Thiboutot M. Dermatol Clin 2000;18:63-71).

\section{H.pylori e fenomeno di Raynaud}

Il fenomeno di Raynaud è un evento frequente e consiste in prolungati episodi di vasospasmo arteriolare che colpisce le estremità e riguarda quasi esclusivamente le giovani donne. Anche se la prevalenza dell' $H p$ non differisce in queste pazienti dai controlli sani, gli unici due studi di terapia eradicante fin qui pubblicati riportano concordemente una riduzione nella frequenza o severità degli episodi morbosi nel 70-90\% dei casi trattati. Il beneficio clinico sarebbe da correlare ad una ridotta produzione di citochine e prostaglandine batteriche ad azione vasoattiva (Gasbarrini A et al. Int J Angiol 1998;7:307-9 ;Csiki Z et al. Orv Hetil 2000;141:2827-9).

\section{H.pylori e Artrite reumatoide}

Esiste un solo studio prospettico, controllato (peraltro di un gruppo italiano) ma ben condotto e con follow-up di 24 mesi (Zentilin $\mathrm{P}$ et al: Aliment Pharmacol Ther 2002;16:1291-9).In questo studio 58 pazienti adulti furono suddivisi in due gruppi: 28 pazienti risultati $\mathrm{Hp}$ + furono sottoposti all'eradicazione mentre gli altri 30 pazienti risultarono fin dall'inizio $H p$-. I pazienti eradicati dimostrarono un progressivo miglioramento degli indici di malattia nel corso del tempo rispetto agli Hp- $(\mathrm{p}<0.0001)$. A distanza di due anni sia gli indici clinici che tutti i principali indici laboratoristici di malattia si presentavano significativamente migliorati negli eradicati. Questo studio supporta la possibilità che l'infezione sia correlata allo sviluppo e al decorso della malattia nei soggetti infetti. E' stato anche osservato che i pazienti con AR e infezione da $H p$ possiedono livelli abnormemente alti di anticorpi diretti verso la proteina batterica HSP65, antigene estremamente simile alle proteine HSP umane. Ciò comincia a chiarire la natura del movimento autoanticorpale che l' $\mathrm{Hp}$ innesca in questa, come probabilmente anche in altre malattie autoimmuni umane. (Kalabay $\mathrm{L}$ et al. Helicobacter 2002;7:250-6).

\section{H.pylori e cefalea emicranica}

I pochi dati fin qui disponibili si possono così riassumere:La prevalenza dell'infezione nei soggetti con emicrania è la stessa che nei controlli sani ma le forme con aura si associano ai ceppi virulenti $\mathrm{Cag} \mathrm{A}+$ più frequentemente di quelle senza aura. (Gasbarrini A et al. Cephalalgia 2000;20:561-5).

Nell'unico trial terapeutico disponibile e relativo a 30 pazienti, l'eradicazione dell' $H p$ ha determinato la completa scomparsa della cefalea nel 17\% dei pazienti ed una riduzione della frequenza, intensità $\mathrm{o}$ durata delle crisi cefalalgiche in un restante $69 \%$ di pazienti. (Gasbarrini A et al. Int J Angiol 1998;7:310-2).

\section{H.pylori e glaucoma}

Persino il glaucoma ad angolo aperto è stato associato all' $H p$. In un recente studio su 41 soggetti, la prevalenza dell'infezione è risultata dell' $88 \%$ versus il 47\% dei controlli. Dopo la terapia antibiotica i principali parametri di malattia ( pressione media intraoculare e campo medio di visione) sono risultati significativamente migliorati nei pazienti eradicati, ma non negli altri pazienti. Questi risultati si sono mantenuti invariati per tutta la durata del follow-up di 2 anni. (Kountouras J et al. Arch Int Med 2002;162:123744).

La possibile relazione causale tra l' $H p$ e questa malattia resta al momento ignota.

L'elenco delle malattie in qualche modo correlate all'Helicobacter resta ancora lungo. Per molte di queste comunque le evidenze sono ancora frammentarie e iniziali ma le prospettive di ricerca in questo campo risultano a dir poco sconfinate.

Come avete visto dunque l'evoluzione delle conoscenze sul rapporto fra Helicobacter e patologia umana è stato in questi anni sorprendente e le prospettive di ricerca futura lo sono ancora di più. Anche se molte delle evidenze sin qui accumulate richiedono conferma, l'Helicobacter si sta rivelando sempre più come un patogeno a tutto campo il cui fardello clinico ed economico nella popolazione mondiale appare enorme. L'infezione ha però tutte le caratteristiche per essere sconfitta. E ancora una volta, perciò, dobbiamo ringraziare i suoi scopritori, Robin Warren e Barry Marshall, per averci aperto la porta su di un universo di nuove conoscenze i cui confini appaiono a tutt'oggi ancora indelimitabili. 